A. Pramesh Rao, G. Swarup and Gopal-Krishna, eds.

\title{
The $74 \mathrm{MHz}$ System on the VLA
}

\author{
R. A. Perley
}

National Radio Astronomy Observatory, P.O. Box 'O', Socorro, NM 87801, USA

W. C. Erickson

U. of Tasmania, School of Math. \& Physics, G.P.O. Box 252-21, Hobart, Tasmania 7001, Australia

N. E. Kassim

Naval Research Laboratory, Remote Sensing Division, Code 7213, Washington, DC 20375-5351, USA

\begin{abstract}
The NRL and the NRAO have jointly implemented a low frequency capability on the Very Large Array. Operating at $73.8 \mathrm{MHz}$, this new frequency band offers unprecedented sensitivity $(\sim 25 \mathrm{mJy} /$ beam $)$ and resolution $\left(\sim 25^{\prime \prime}\right)$. This paper briefly describes the this new receiving system, and the methodology for using it for astronomy.
\end{abstract}

\section{Introduction - Low Frequencies on the VLA}

The original design of the Very Large Array included only four frequency bands, centered near $21,6,2$ and $1.3 \mathrm{~cm}$ wavelength. However, there is no fundamental reason a low frequency system could not operate on the array - the principles of aperture synthesis are as applicable to $100 \mathrm{MHz}$ as they are to $5 \mathrm{GHz}$, and the key components of the array - the signal collection (antennas), signal transmission (waveguide), and signal processing (correlator and post-processing) are also essentially frequency independent. Thus, as soon as the construction phase of the VLA ended, discussions on implementing a low frequency capability began.

The initial low-frequency system was installed at P-band ( 300 to $340 \mathrm{MHz}$ ) between 1983 and 1989. It is a prime-focus system, as it is impractical to implement a secondary focus system at such a long wavelength. The feed is a crossed dipole, situated in front of the Cassegrain subreflector, which thus acts as a (rather imperfect) subreflector. Because of this, and because the phase center is located approximately $50 \mathrm{~cm}$ in front of the true focus, this system has both a low efficiency (less than $40 \%$ ) and a very broad shoulder of width $\sim 12^{\circ}$ in the antenna power pattern. Nevertheless, it has been a very successful and widely used frequency band at the VLA. Most importantly, it encouraged the development of the multi-faceted imaging algorithms needed for low frequency observing, as described later in this paper. 


\section{The $74 \mathrm{MHz}$ System on the VLA - The Trial Array}

The success of the $327 \mathrm{MHz}$ system soon led to consideration of a lower frequency facility. Perley and Erickson (1984) published a memorandum advocating a free-standing array which would make use of the VLA's infrastructure (most importantly, the waveguide transmission system) to enable an array with $\sim 25^{\prime \prime}$ resolution. However, no source of funding was obvious, and it was subsequently decided that a trial system, comprising a simple feed system on a few of the VLA's antennas, would suffice to answer the key questions of calibration and imaging of low frequency, long-baseline data.

The feed system chosen is essentially the same as that used at $327 \mathrm{MHz}-$ crossed dipoles in front of the subreflector. Because of the long wavelength, the defocussing errors which severely affect $327 \mathrm{MHz}$ performance are not serious at $74 \mathrm{MHz}$. However, because the antenna itself is only $\sim 6 \lambda$ in diameter, and because of the profound effect of the antenna quadrupod structure, it was anticipated that the forward gain and sidelobe structure would be fairly poor as subsequent measurements have borne out.

Progress with this trial system was glacial - tests began in late 1984 (initially done with a commercial Yagi antenna, mounted on the side of the 25-meter reflector!), and culminated in 1991 with eight antennas outfitted with the systems described above. Observations with this trial array were very successful, and permitted us to define the data calibration and imaging methodologies. Initial results were published by Kassim et al. (1993)

\section{The 74 MHz System on the VLA - The Full Array}

Despite its early successes, it proved remarkably difficult to expand the 8antenna trial array to a full 27-antenna system. This was a time of declining monies for research equipment for the VLA, and avilable resources were primarily devoted to upgrading the 21-cm system. This situation changed in 1997, when Bill Erickson offered to design and build the needed receivers, and the NRL agreed to foot the costs. The NRAO agreed to provide the dipoles and local engineering support.

Because of concerns about blockage at higher frequencies, a deployable crossed-dipole feed was designed and employed. Tests show that this system decreases system performance by about $6 \%$ at $21 \mathrm{~cm}$, and by smaller amounts at higher frequencies. The mounting system is very simple - two ropes, each of which supports one dipole, are threaded through eyebolts located on opposite quadrupod legs at the appropriate height. The ends of these ropes are tied to cleats located at a convenient height on the quadrupod legs. The signal cables drop about 7 meters to the antenna surface, where they pass through the roof of the vertex room to the amplifiers.

The amplifiers and feeds were built over the summer of 1997, and deployed during the fall of that year. By October there were 15 antennas operational, and the full array was outfitted in January 1998. The initial imaging results were spectacular, with images of large and complex objects which are much superior to those generated by the trial system. Figure 1 shows two examples - Aconfiguration images of Virgo A (3C274), and Cassiopeia A (3C461) made with 

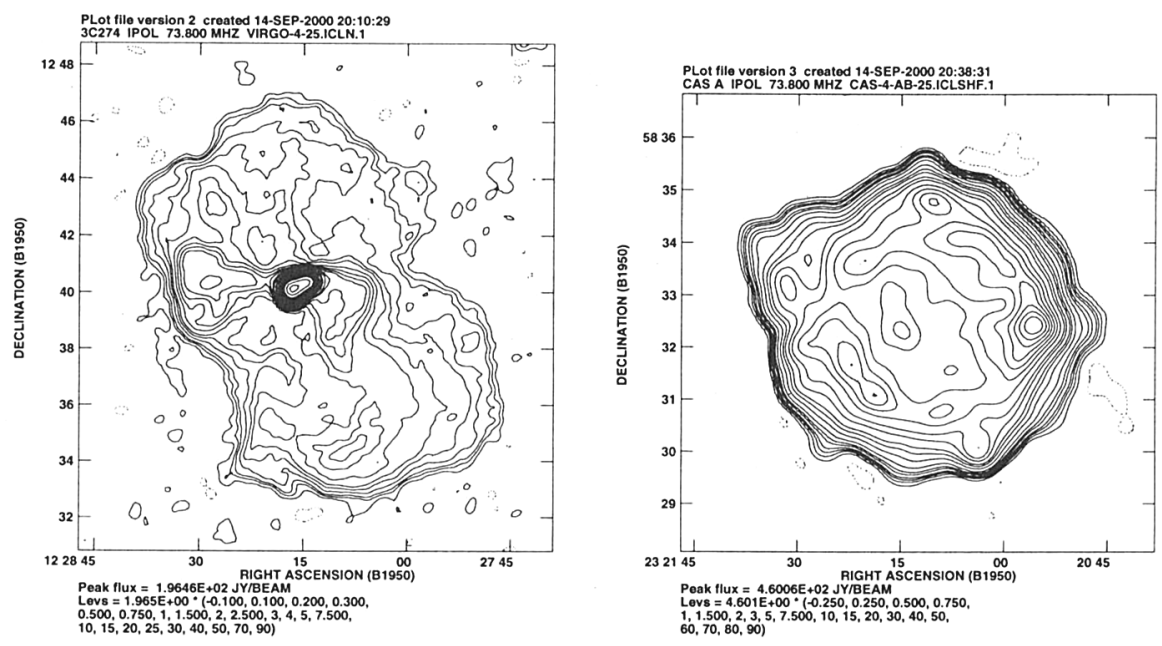

Figure 1. Radio Images of Virgo A (left) and Cassiopeia A (right), made with the $74 \mathrm{MHz}$ system of the VLA. The resolution is $25^{\prime \prime}$, and the dynamic range is about 1000:1

about an hour's data with the completed $74 \mathrm{MHz}$ system. Since both of these objects are very bright, calibration and imaging were very straightforwards, with needing the special techniques described later in this paper.

\section{Performance Characteristics of the $74 \mathrm{MHz}$ System}

The performance characteristics of the VLA's $74 \mathrm{MHz}$ system are listed in Table 1 . Note that the quoted system temperature is appropriate for the galactic caps - values for fields near the galactic plane will be two to three times higher, and perhaps ten times greater for fields near the galactic center. The sensitivity listed is typical for the $\mathbf{A}$ and $\mathbf{B}$ configurations, for regions away from the galactic plane. Sensitivies for the shorter configurations are considerably poorer - presumably due to low-level, broad-band emissions from the antennas and equipment located at the VLA site.

Table 1. Performance Characteristics of the VLA $74 \mathrm{MHz}$ System

\begin{tabular}{|l|l|}
\hline Center Frequency & $73.8 \mathrm{MHz}$ \\
Bandwidth & $1.6 \mathrm{MHz}$ \\
Primary Beamwidth (FWHM) & 11.7 \\
System Temperature (minimum) & $1500 \mathrm{~K}$ \\
Efficiency & $15 \%$ \\
Sensitivity (A and B configs.) & $\sim 25 \mathrm{mJy} /$ beam in $8 \mathrm{hr}$ \\
Resolution (A config.) & $25^{\prime \prime}$ \\
\hline
\end{tabular}




\section{Calibration of $74 \mathrm{MHz}$ Data}

Observations taken with the trial $74 \mathrm{MHz}$ system clearly demonstrated that the methodologies and algorithms which had been developed for calibration at the standard VLA frequencies were generally sufficient for $74 \mathrm{MHz}$, and only minor modifications and additions have been necessary. The key steps in calibration and imaging at these very low frequencies are described below. These steps are described in much more detail in a tutorial written by Joseph Lazio of the NRL. The tutorial will be found at: http://rsd-www.nrl.navy.mil/7213/lazio/tutorial. Also located here are the models of the prominent calibrator sources Cygnus $\mathrm{A}$, Cassiopeia A, Taurus A and Virgo A.

\subsection{RFI Excision}

There is virtually no external RFI within our passband of $73.0-74.6 \mathrm{MHz}$. Essentially all non-astronomical signals are internally generated by the VLA's antenna electronics and (to a lesser degree) signals emanating from sources on the VLA site. By far the most common is the $100 \mathrm{kHz}$ comb generated by the VLA's monitor and control system. The oscillators responsible for this are located in every antenna, and they are sufficiently similar to result in spurious correlation, especially between certain pairs of antennas. The net (averaged over frequency) spurious visibility is not great - a few tens of Jy - and can be effectively removed in the spectral domain.

The AIPS program FLGIT written by Eric Greisen 'shaves' spectral channels whose flux density exceeds a threshhold level which can either be set by the user, or can be determined by statistical analysis of channels declared to be free of the undesired effect. This elementary approach has proven to be very effective in removing weak RFI. Because the VLA's correlator can only resolve the passband into $24-\mathrm{kHz}$ channels in the observing mode typically employed, the application of FLGIT can remove a significant fraction of the data - typically 10 to $25 \%$. It is to be hoped that any future correlator will provide considerably more channels with higher frequency resolution, allowing much more precise removal of these artificial signals.

\subsection{Bandpass Calibration}

The need to remove internally-generated RFI requires use of the spectral line system. Before the spectra can be 'shaved' by FLGIT, the bandpass shapes (and phase gradients due to delay errors) must be removed by bandpass calibration. Fortunately, this can be done almost trivially by observations of Cygnus A, since the flux density of that object is nearly always much greater than the intensity of the RFI. A good model of this source is required, and is available from the NRL's website noted above.

\subsection{Amplitude Calibration}

At this frequency, there is only one good calibrator - Cygnus A. Because it is partially resolved, a good model is required for accurate amplitude and phase calibration. This has been generated by multiple observations, and is available at the NRL website. We base our flux scale on the Baars et al. value of $17086 \mathrm{Jy}$. Although Cygnus A is the best amplitude calibrator, others can be employed 
if necessary. Virgo A is acceptable in all configurations, while Taurus A and Cassiopeia A can be used in the smaller configurations. All of these sources are significantly resolved, so model images are necessary for calibration. These can be obtained from the NRL website listed above.

Accurate flux density determination depends on the antennas having stable, known gain. Because the VLA's digital correlator requires a constant amplitude input, the antenna gains will vary by a factor depending on the input power - determined primarily by the galactic background as seen by the antenna main beam. The observed correlation is thus modified by this factor, which can be removed after correlation provided it has been accurately measured. The accuracy of a flux density determination will then depend on the accuracy of this gain modification. We have tested this by repeated observations of strong $3 \mathrm{C}$ sources in the $\mathbf{A}, \mathbf{B}$, and $\mathbf{C}$ configurations. Our results show that flux density determinations should be reliable to better than $5 \%$.

\subsection{Phase Calibration - the Role of Self-Calibration}

It is not generally possible to use Cygnus $A$ to provide useful phase calibration for data taken in the $\mathbf{A}$ or $\mathbf{B}$ configurations, due to large and rapid phase variations primarily caused by ionospheric travelling waves. An example of the amplitude and time scale of these variations is shown in Figure 2. Because of their long wavelength (hundreds of kilometers or more), and distance $(400 \mathrm{~km})$, we expect that the interferometer phase error will be highly correlated over spatial scales of tens of kilometers (as is clearly shown in Figure 2), and over angular scales of many degrees - thus permitting phase self-calibration using emission within the primary beam itself. An elementary calculation shows that the total flux density within the VLA's primary beam exceeds $700 \mathrm{Jy}$ to the noise level of an 8 hour integration. It should only be necessary to know a priori the location and strength of the strongest few objects to enable successful self-calibration.

Experiments with the full 27-antenna array have shown that this simple expectation is indeed realized. In fact, in most cases experiments have shown it is only necessary to know the location of the stongest objects totaling $50 \mathrm{Jy}$ in flux to enable successful phase calibration. This is an amazingly easy constraint to meet, for nearly every randomly-picked field of view will contain at least one $3 \mathrm{C}$ object (or equivalent at southern declinations) whose flux density is close to this minimum value. In general, existing all-sky surveys at 327 or $1400 \mathrm{MHz}$ (i.e., the NVSS, WENSS, and SUMSS surveys) will identify the strongest objects totalling at least $100 \mathrm{Jy}$.

\section{Imaging at Low Frequencies}

Here we summarize the special procedures needed for effective imaging at these low frequencies. Overall, there is nothing unique about these procedures - they are basic extensions of techniques developed for higher frequencies. However, due to the effects described below, whereas at higher frequencies one often does not have to employ these special methods, at the low frequency of $73.8 \mathrm{MHz}$, they must nearly always be used. 


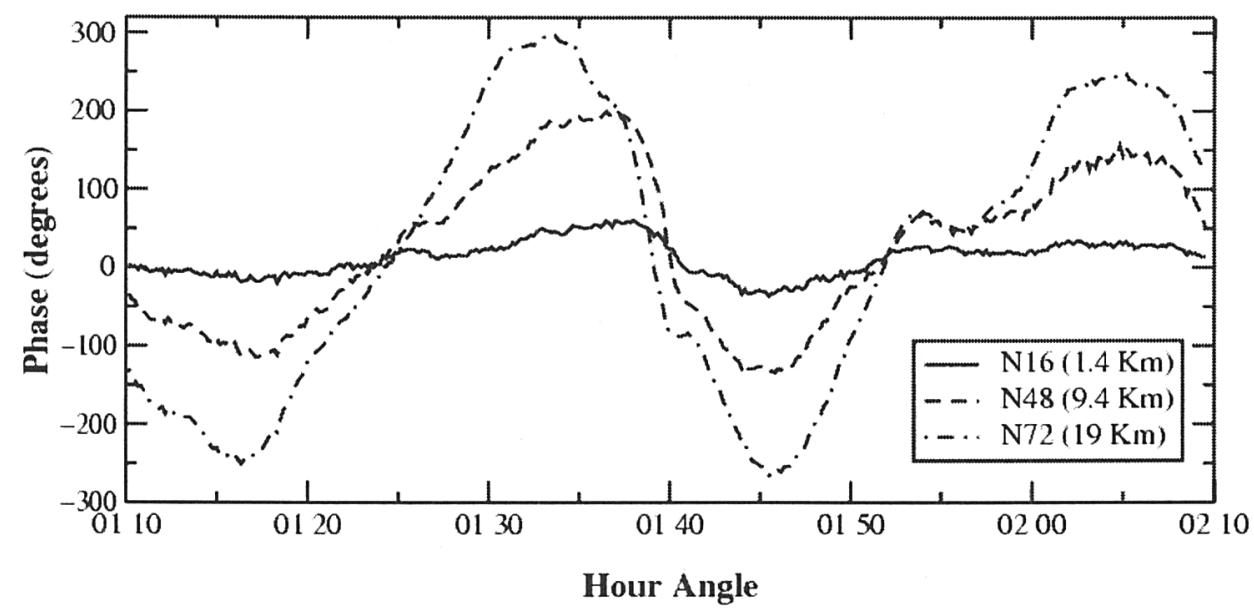

Figure 2. The ionospheric phase towards Cygnus A, as seen by three antennas on the north arm, referenced to the center of the array. The ionospheric phase is closely proportional to distance over the $19 \mathrm{~km}$ extent of this arm, demonstrating the high degree of correlation between adjacent antennas in normal observing conditions.

\subsection{Confusion and Sidelobes}

Diffraction is ultimately responsible for the particular problems experienced in low frequency imaging. Because of the long wavelengths, and our small (in wavelengths) aperture, the primary beam is both very large, and has high sidelobes. Since the receiver noise is a tiny fraction of the total signal, measureable coherence is detected from objects all over the sky entering the system through the sidelobes. This situation contrasts with that at centimeter wavelengths, where the combination of noisy receivers (compared to the sky temperature) and good sidelobes ensures that one need not be concerned with out-of-beam emission in most cases.

Because of this 'signal-enriched' environment, it is necessary in nearly all cases to image the entire primary beam, and to target the brightest objects in the sidelobes, wherever they are in the sky. Failure to do this will result in uncleaned sidelobes of these objects remaining in the image of the object of interest.

\subsection{Non-Coplanar Baselines}

The standard two-dimensional FFT inversion of visibility data to obtain an image is only strictly correct for observations taken with coplanar arrays. For VLA data taken over any significant hour angle range, a three-dimensional inversion, or multiple small two-dimensional inversions, each with different geometry, is necessary if the maximum baseline exceeds $B \gtrsim D^{2} / \lambda$. For details and examples, see Perley (1999), and Cornwell and Perley (1992). The latter method is now well established, and is implemented under AIPS in the task IMAGR. 
The extra computational load is significant. However, current desktop machines can handle the VLA's B configuration data easily — and extrapolations of Moore's law gives us confidence that even the much more demanding $\mathbf{A}$ configuration will soon be easily accommodated.

\subsection{The Isoplanatic Patchsize}

As shown in Figure 2, the long-wavelength ionospheric waves result in a phase error approximately proportional to distance. However, even through these waves are long, they are not long enough to ensure that the phase differential between two widely-separated antennas remains independent of angle. Indeed, we must expect the interferometer phase to vary significantly over the $\sim 10^{\circ}$ angles of the primary beam, for baselines longer than a few kilometers. The angle over which a particular phase solution is valid for imaging is termed the 'isoplanatic angle'. Observations made at $74 \mathrm{MHz}$ with the VLA clearly show that even under benign conditions in $\mathbf{B}$ configuration, bright objects separated by more than a few degrees show different degrees of distortion - a sure sign of phase errors.

No 'single-angle' solution self-calibration procedure can successfully remove these effects, which are, in effect, caused by curvature in the phase screen above the array. Future software must enable variable-angle phase calibration to enable good low frequency, high fidelity imaging.

\subsection{Multi-Field Imaging with IMAGR}

The arguments above demonstrate that low frequency observations must be taken in spectral line mode to enable editing and wide-field imaging, and that the imaging must be done through many small and overlapping sub-fields. All the editing, calibration, and imaging, with the exception of angle-variant selfcalibration, can be accomplished with the existing AIPS package. Shortly, all necessary operations, including the angle-variant calibration, will be available within aips++.

At this time, the AIPS program IMAGR is well suited for imaging these low frequency data. An example of a deep integration of the Coma cluster, made with the VLA in its $\mathbf{A}$ and $\mathbf{B}$ configurations, and imaged by IMAGR is shown in Figure 3. The total flux density detected exceeds $450 \mathrm{Jy}$, and the phase calibration was begun using 3C277.3 and 3C286 alone. Both objects (totaling about $50 \mathrm{Jy}$ ) are within the primary beam.

\section{The Future of the $74 \mathrm{MHz}$ System}

The $74 \mathrm{MHz}$ system is a trial system, and will only be mounted for short periods of time during which all accumulated proposals will be scheduled. The Expanded VLA Project has plans for modifying the quadrupod so that low frequency feeds could be mounted at the prime focus. This should at least double the sensitivity of this band. These modifications are a part of Phase II of the project, for which a proposal is being developed.

However - despite the success of this low frequency system, the sensitivity is fundamentally limited by the VLA's modest collecting area. Even if the VLA 


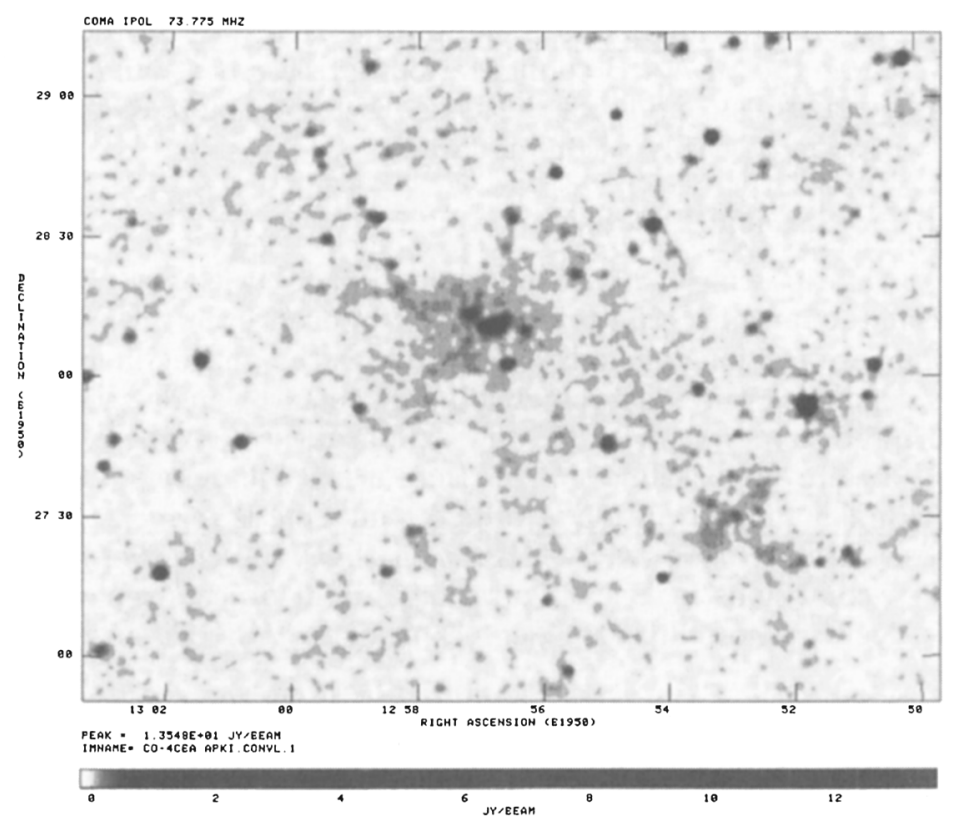

Figure 3. A $74 \mathrm{MHz}$ image of the Coma region with $25^{\prime \prime}$ resolution, made with about 8 hours of VLA data, and using local self-calibration for removing the ionospheric phase errors. The rms noise is about 25 $\mathrm{mJy} /$ beam.

could be made $50 \%$ efficient, and the bandwidths significantly increased, it is not possible for the EVLA - or the GMRT - to be more sensitive than $\sim 1$ $\mathrm{mJy} /$ beam at $74 \mathrm{MHz}-100$ times less sensitive than at $20 \mathrm{~cm}$ to normalspectrum objects. The only solution to this is by building a system with much greater collecting area. The LOFAR project seeks to accomplish this.

Basic research in radio astronomy at the Naval Research Laboratory is supported by the office of Naval Research. The National Radio Astronomy Observatory is a facility of the Nastional Science Foundation operated under cooperative agreement by Associated Universities, Inc.

\section{References}

Cornwell, T.J., and Perley, R.A. 1992, A\&A, 261, 353.

Kassim, N.E., Perley, R.A., Erickson, W.C., \& Dwarakanath, K.S. 1993 AJ106, 2218.

Perley, R.A. 1999, in ASP Conference Series Vol. 180, Synthesis Imaging in Radio Astronomy II, ed. G.B. Taylor, C.L. Carilli \& R.A. Perley. (San Francisco: ASP), 383.

Perley, R.A., and Erickson, W.C. 1984. VLA Scientific Memorandum \#146. 